\title{
Relationship between Socioeconomic Status and Prevalent Prostate Cancer in the South Korea
}

\author{
Hee-Won Hur ${ }^{1}$, So-Yeon Ryu ${ }^{2}$, Jong Park ${ }^{2}$, Seong-Woo Choi ${ }^{2 *}$
}

\begin{abstract}
Background: Prostate cancer prevalence recently has increased among male adults in South Korea. But, few study has evaluated the reason. Therefore, we investigated the relationship between socioeconomic status and prevalent prostate cancer. Methods: This study enrolled 16,215 males aged 40 years and over who took part in the Korean National Health and Nutrition Examination Survey 2007-2016. In addition, we obtained the 2000-2016 age-standardized incidence rate and age-standardized mortality rate of prostate cancer from the Korean Statistical Information Service. Results: After adjusting for other covariates, prevalent prostate cancer was significantly associated with monthly household income (OR 3.71, 95\% confidence interval [CI] 1.48-9.30, for highest vs. lowest) and significantly associated with education level (OR 3.66, 95\% CI 1.54-8.70, for $\geq 13$ vs. $\leq 6$ ). In the analysis of the age-standardized incidence rate and the age-standardized mortality rate, the age-standardized incidence rate has soared from 2000 to 2011 and then decreased gradually, but the age-standardized mortality rate did not change. Conclusion: In our results, prevalent prostate cancer increased significantly with socioeconomic status and the increase in prevalent prostate cancer may be attributable to earlier detection rather than to a real increase in prevalence.
\end{abstract}

Keywords: Prostatic neoplasms- social class- income- educational status

Asian Pac J Cancer Prev, 20 (10), 3137-3144

\section{Introduction}

Globally, prostate cancer is the most common male cancer (Global Cancer Observatory, 2018) and the number of male patients diagnosed with prostate cancer during the year 2012 was about 1.1 million, and about $70 \%$ of prostate cancer cases occurred in developed countries (Global Cancer Observatory, 2018). In the United States, the incidence of prostate cancer peaked in 1992 and subsequently declined, but the prostate cancer incidence is still the highest in American men (Cronin et al., 2018). The new case of prostate cancer in Korea was 3,487 in 2005 (Jung et al., 2009) and increased about 3.4 times to 11,800 in 2016 (Jung et al., 2019).

Age has been known to be the most essential risk factor for prostate cancer; the incidence increases with age after 50 years (Kim, 2004). Also, the incidence of prostate cancer is higher in blacks than in whites, with early onset and malignancy, leading to higher mortality rates (Schwartz et al., 2003). However, none of the risk factors for prostate cancer are clearly known except for age, race, and family history (Platz and Giovannucci, 2006). A westernized lifestyle, obesity, lack of exercise and activity have been reported as risk factors, but the results have been inconsistent (Perez-Cornago et al., 2017; Malik et al., 2018).
Socioeconomic status (SES) is related to health. The lower the SES is, the lower the self-reported health (Borg and Kristensen, 2000) and the increased incidence of illness and mortality (Saydah et al., 2013). However, the relationship between socioeconomic level and cancer incidence and mortality is unclear, and prostate cancer studies have shown varying results. In some studies, the incidence of prostate cancer increased with SES (Cheng et al., 2009), while in other studies it decreased with a higher SES (Baquet et al., 1991), and still other studies found no association between SES and prostate cancer (Williams and Horm, 1977; Mackillop et al., 2000).

However, most of these studies have been performed in Western populations and few studies have been conducted in Korea, where prostate cancer has soared recently. Specifically, few study has evaluated whether prostate cancer in Korea has actually increased because of a specific cause or as a product of early detection. For thyroid cancer, which showed a similar surge, many experts warned that overdiagnosis should be suspected (Choi et al., 2013; Ahn et al., 2014).

Therefore, this study assessed the association of prevalent prostate cancer with SES using the following two data sets: 1) the 2007-2016 Korea National Health and Nutrition Examination Survey (KNHANES), 2) the 
prostate cancer age-standardized incidence rate (AIR) using cancer registration statistics, and the prostate cancer age-standardized mortality rate (AMR) using cause-of-death statistics released by the Korean Statistical Information Service (KOSIS).

\section{Materials and Methods}

\section{Subjects}

This study used the 2007-2016 KNHANES data on SES, the prevalent case of prostate cancer, and health behaviors and 2000-2016 KOSIS data on the AIR and AMR of prostate cancer. The details of KNHANES are already demonstrated in previous publication (Kweon et al., 2014). The Korea Centers for Disease Control and Prevention (KCDCP) annually conducts the KNHANES using a sampling design to produce health statistics representative of residents of the Republic of Korea. The KNHANES consisted with the health and nutrition interview and health examination. The health and nutrition interview are conducted by trained interviewers using questionnaires, and the health examinations are performed by trained medical staff. The 2007-2016 surveys included 83,503 participants. After excluded 44,501 women, 19,216 people under 40, and 3,571 people without income, education, or prostate cancer screening data, we analyzed 16,215 men aged 40 years or older.

\section{Measurements}

Trained investigators interviewed the subjects individually using a questionnaire. A person who answered 'yes' to a question about being diagnosed with prostate cancer was defined as a patient with prostate cancer. Monthly household income was classified into quartiles. Education level was divided into $\leq 6,7-9,10-12$, and $\geq$ 13 years. BMI was presented by dividing the weight in kilograms by the square of the height in meters. Marriage status was classified as unmarried and married; residence area was divided into urban and rural areas. Current smoking was defined as people who smoked or smoked occasionally, and monthly drinking was defined as having one or more drinking experiences during the previous month. Physical activity was defined as walking for more than thirty minutes at one time and more than 5 times per week. Health checkup in the previous 2 years was defined as a case in which a health checkup had been conducted in the past 2 years; cancer examination in the prior 2 years was defined similarly. Hypertension was defined as taking a hypertensive medicine or blood pressure above 140/90 $\mathrm{mmHg}$; diabetes mellitus was defined as taking a diabetes medicine or using insulin, or fasting blood glucose above $126 \mathrm{mg} / \mathrm{dL}$. Dyslipidemia was defined as taking a dyslipidemic medicine or one of the following four: total cholesterol above $240 \mathrm{mg} / \mathrm{dL}$, triglycerides above $200 \mathrm{mg} /$ $\mathrm{dL}$, low-density lipoprotein cholesterol above $160 \mathrm{mg} / \mathrm{dL}$, and high-density lipoprotein cholesterol below $40 \mathrm{mg} /$ dL. Cardiovascular disease was defined as having been diagnosed with myocardial infarction or angina pectoris or stroke. Other cancer was defined as having been diagnosed with a cancer other than prostate cancer.

\section{The AIR and AMR of prostate cancer}

The AIR and AMR of prostate cancer in 2000-2016 were analyzed using cancer registration and cause-of-death statistics released on the KOSIS web page (Korean Statistical Information Service, 2018).

\section{Statistical analysis}

The survey responses were weighted based on a multilevel, multiple, probability sampling design to represent for nationally representative prevalence estimates of the Korean population. The estimates were calculated with consideration for the primary sampling unit, stratification variables, and sampling weights. Data were expressed as estimated percentages (standard errors [SEs]) or mean \pm standard deviation. The distributions of each variable according to the quartiles of monthly household income and education level were analyzed using the analysis of variance. The associations of prevalent prostate cancer with the quartiles of monthly household income and education level were analyzed using a multivariate logistic regression analysis. Model 1 was adjusted for age, BMI, survey year, marital status, and residence area. Model 2 was additionally adjusted for current smoking, monthly drinking, physical activity, health checkup in the prior 2 years, cancer examination in the prior 2 years, hypertension, diabetes, dyslipidemia, cardiocerebrovascular disease, and other cancer. Model 3 was additionally adjusted for education level or monthly household income. A P-value $<0.05$ was considered statistically significant. Statistical analysis was performed using SPSS ver. 18.0.

\section{Ethics statement}

This study was conducted according to the Declaration of Helsinki and all subjects provided informed consent for their data use. The KCDCP ethics committee approved the study protocol (2007-02CON-04-P, 2008-04EXP-01-C, 2009-01CON-03-2C, 2010-02CON-21-C, 2011-02CON06-C, 2012-01EXP-01-2C, 2013-07CON-03-4C, 2014-12EXP-03-5C, 2015-01-02-6C).

\section{Results}

\section{General characteristics of the subjects}

Fifty-eight patients were diagnosed with prostate cancer. Their mean age was $55.0 \pm 0.1$ years, and their mean BMI was $24.2 \pm 0.0 \mathrm{~kg} / \mathrm{m}^{2}$. The lowest to highest quartiles of monthly household income contained 17.3, $24.9,27.4$, and $30.4 \%$, respectively. The education level was $\leq 6$ for $19.9 \%, 7-9$ years for $15.0 \%, 10-12$ years for $34.1 \%$, and $\geq 13$ years for $31.0 \%$ (Table 1 ).

\section{Subject characteristics by quartiles of monthly household income}

Age, BMI, survey year, education level, marital status and smoking differed significantly according to the quartiles of monthly household income. In addition, urban dwellers, monthly household income, health checkup and cancer examination in the prior 2 years, and dyslipidemia significantly increased with increasing monthly household income, while current smoking, 
Table 1. General Characteristics of the Subject

\begin{tabular}{|c|c|c|}
\hline Variables & Number & $\begin{array}{l}\mathrm{e} \%(\mathrm{SE}) \text { or } \\
\text { Mean } \pm \mathrm{SD}\end{array}$ \\
\hline Total & 16,215 & \\
\hline Prostate cancer patients & 69 & $0.3(0.0)$ \\
\hline Age (year) & & $55.1 \pm 0.1$ \\
\hline $40-49$ & 4,389 & $37.6(0.5)$ \\
\hline $50-64$ & 6,292 & $41.8(0.5)$ \\
\hline$\geq 65$ & 5,534 & $20.6(0.4)$ \\
\hline $\operatorname{BMI}\left(\mathrm{kg} / \mathrm{m}^{2}\right)$ & & $24.2 \pm 0.0$ \\
\hline$<18.5$ & 464 & $2.4(0.1)$ \\
\hline $18.5-24.9$ & 9,816 & $58.7(0.5)$ \\
\hline $25.0-29.9$ & 5,480 & $35.7(0.4)$ \\
\hline$\geq 30$ & 447 & $3.2(0.2)$ \\
\hline \multicolumn{3}{|l|}{ Survey year } \\
\hline 2007 & 791 & $4.7(0.5)$ \\
\hline 2008 & 1,779 & $9.8(0.6)$ \\
\hline 2009 & 2,114 & $10.4(0.7)$ \\
\hline 2010 & 1,807 & $10.4(0.8)$ \\
\hline 2011 & 1,791 & $10.7(0.8)$ \\
\hline 2012 & 1,635 & $10.5(0.7)$ \\
\hline 2013 & 1,537 & $10.5(0.7)$ \\
\hline 2014 & 1,442 & $10.3(0.7)$ \\
\hline 2015 & 1,566 & $10.8(0.7)$ \\
\hline 2016 & 1,753 & $12.1(0.4)$ \\
\hline \multicolumn{3}{|l|}{ Monthly household income } \\
\hline Lowest & 3,641 & $17.3(0.4)$ \\
\hline Medium-lowest & 4,125 & $24.9(0.5)$ \\
\hline Medium-highest & 4,044 & $27.4(0.5)$ \\
\hline Highest & 4,405 & $30.4(0.6)$ \\
\hline \multicolumn{3}{|l|}{ Education (year) } \\
\hline$\leq 6$ & 4,131 & $19.9(0.4)$ \\
\hline $7-9$ & 2,572 & $15.0(0.4)$ \\
\hline $10-12$ & 5,033 & $34.1(0.5)$ \\
\hline$\geq 13$ & 4,479 & $31.0(0.6)$ \\
\hline \multicolumn{3}{|l|}{ Marital status } \\
\hline Single & 1,888 & $13.6(0.4)$ \\
\hline Married & 14,316 & $86.4(0.4)$ \\
\hline \multicolumn{3}{|l|}{ Residential area } \\
\hline Urban & 11,953 & $77.8(1.0)$ \\
\hline Rural & 4,262 & $22.2(1.0)$ \\
\hline Current smoking & 7,768 & $49.4(0.5)$ \\
\hline $\begin{array}{l}\text { Alcohol intake in past } \\
\text { month }\end{array}$ & 12,562 & $80.5(0.4)$ \\
\hline Physical activity ${ }^{\mathrm{a}}$ & 6,796 & $39.9(0.5)$ \\
\hline $\begin{array}{l}\text { Health checkup in the prior } \\
2 \text { years }\end{array}$ & 11,215 & $68.7(0.5)$ \\
\hline $\begin{array}{l}\text { Cancer examination in the } \\
\text { prior } 2 \text { years }\end{array}$ & 9,375 & $56.4(0.5)$ \\
\hline Hypertension $^{\mathrm{b}}$ & 7,347 & $41.9(0.5)$ \\
\hline Diabetes $^{\mathrm{c}}$ & 2,821 & $16.1(0.3)$ \\
\hline Dyslipidemia $^{\mathrm{d}}$ & 2,695 & $16.6(0.3)$ \\
\hline
\end{tabular}

Relationship between Socioeconomic Status and Prostate Cancer

Table 1. Continued

\begin{tabular}{lcc}
\hline Variables & Number & $\begin{array}{c}\mathrm{e} \%(\mathrm{SE}) \text { or } \\
\text { Mean } \pm \text { SD }\end{array}$ \\
$\begin{array}{l}\text { Cardiocerebrovascular } \\
\text { disease }\end{array}$ & 1,257 & $5.9(0.2)$ \\
Other cancer & 663 & $3.3(0.2)$ \\
\hline
\end{tabular}

All values are presented are given as number and estimated percentage(standard error) or mean \pm standard deviation; $\mathrm{e} \%$, estimated percentage; SE, standard error; SD, standard deviation; a ,Physical activity was indicated as 'yes' when the subject walked for more than $30 \mathrm{~min}$ at a time and more than five times per week; ${ }^{\mathrm{b}}$, Hypertension was defined as systolic blood pressure $\geq 140 \mathrm{mmHg}$ or diastolic blood pressure $\geq 90 \mathrm{mmHg}$ or taking antihypertension medication; ${ }^{\mathrm{c}}$, Diabetes was defined as fasting serum glucose $\geq 126 \mathrm{mg} / \mathrm{dL}$ or taking insulin or oral diabetes medication; ${ }^{\mathrm{d}}$, Dyslipidemia was defined as taking a dyslipidemic medicine or total cholesterol $\geq 240 \mathrm{mg} / \mathrm{dL}$ or triglycerides $\geq 200 \mathrm{mg} / \mathrm{dL}$ or low-density lipoprotein cholesterol $\geq 160 \mathrm{mg} / \mathrm{dL}$ or high-density lipoprotein cholesterol $\leq 40 \mathrm{mg} / \mathrm{dL}$.

hypertension, diabetes, cardiocerebrovascular disease, and other cancer significantly decreased with increasing monthly household income (Table 2).

\section{Subject characteristics by education level}

Prevalent case of prostate cancer, age, BMI, survey year, monthly household income, current smoking, monthly drinking, cancer examination in the prior 2 years, and dyslipidemia differed significantly according to the education level. In addition, single person, urban dwellers and health checkup in the prior 2 years increased significantly with education level, while hypertension, diabetes, cardiocerebrovascular disease, and other cancer decreased significantly with increasing education level (Table 3).

Odds ratios (ORs) for prevalent prostate cancer by quartiles of monthly household income and education level

After adjusting for age, BMI, survey year, marital status, residence area, current smoking, monthly drinking, physical activity, health checkup and cancer examination in the prior 2 years, hypertension, diabetes, dyslipidemia, cardiocerebrovascular disease, other cancer, and education level (Model 3), prevalent prostate cancer was significantly associated with monthly household income (OR 3.71, 95\% confidence interval [CI] 1.48-9.30, for highest vs. lowest). When adjusted for the same variables and monthly household income (Model 3), prevalent prostate cancer was significantly associated with education level (OR $3.66,95 \%$ CI $1.54-8.70$, for $\geq 13$ vs. $\leq 6$ ) (Table 4).

\section{The AIR and AMR of prostate cancer}

Figure 1 shows the AIR and AMR of prostate cancer from 2000 to 2016 in South Korea. AIR per 100,000 people increased from 7.3 in 2000 to 28.0 in 2011. It gradually decreased after 2011, reaching 25.5 in 2015 and increased again to 28.2 in 2016 . However, the AMR per 100,000 did not change significantly from 4.2 in 2000 to 5.2 in 2016 .

\section{Discussion}

This study investigated the relationship between 
Table 2. Characteristics of Subjects by Quartiles of Monthly Household Income

\begin{tabular}{|c|c|c|c|c|c|}
\hline \multirow[t]{2}{*}{ Variables } & \multicolumn{4}{|c|}{ Monthly household income } & \multirow[t]{2}{*}{ P-value } \\
\hline & Lowest & Medium-lowest & Medium-highest & Highest & \\
\hline Prostate cancer patients & $0.4(0.1)$ & $0.3(0.1)$ & $0.1(0.1)$ & $0.4(0.1)$ & 0.072 \\
\hline Age (year) & & & & & $<0.001$ \\
\hline $40-49$ & $14.0(0.8)$ & $35.6(1.0)$ & $47.1(1.0)$ & $45.7(1.0)$ & \\
\hline $50-64$ & $31.5(1.0)$ & $43.4(0.9)$ & $41.4(1.0)$ & $47.2(0.9)$ & \\
\hline$\geq 65$ & $54.5(1.0)$ & $21.0(0.6)$ & $11.5(0.5)$ & $7.1(0.4)$ & \\
\hline $\operatorname{BMI}\left(\mathrm{kg} / \mathrm{m}^{2}\right)$ & & & & & $<0.001$ \\
\hline$<18.5$ & $4.9(0.4)$ & $2.6(0.3)$ & $1.9(0.2)$ & $1.0(0.2)$ & \\
\hline $18.5-24.9$ & $63.6(0.9)$ & $59.9(0.9)$ & $56.1(0.9)$ & $57.0(0.9)$ & \\
\hline $25.0-29.9$ & $28.6(0.9)$ & $34.3(0.9)$ & $38.3(0.9)$ & $38.9(0.9)$ & \\
\hline$\geq 30$ & $2.9(0.4)$ & $3.3(0.4)$ & $3.6(0.4)$ & $3.0(0.3)$ & \\
\hline Survey year & & & & & $<0.001$ \\
\hline 2007 & $6.6(0.9)$ & $6.0(0.7)$ & $4.3(0.6)$ & $2.8(0.5)$ & \\
\hline 2008 & $14.1(1.2)$ & $11.3(0.9)$ & $9.9(0.9)$ & $5.5(0.7)$ & \\
\hline 2009 & $13.3(1.1)$ & $11.5(0.8)$ & $10.3(0.9)$ & $7.7(0.8)$ & \\
\hline 2010 & $9.1(0.9)$ & $11.3(1.0)$ & $11.6(1.0)$ & $9.3(0.9)$ & \\
\hline 2011 & $9.5(0.9)$ & $10.5(0.9)$ & $11.0(1.0)$ & $11.2(1.0)$ & \\
\hline 2012 & $9.3(1.0)$ & $10.9(1.1)$ & $11.2(0.9)$ & $10.2(1.0)$ & \\
\hline 2013 & $9.7(1.0)$ & $9.9(0.8)$ & $11.4(0.9)$ & $10.9(1.0)$ & \\
\hline 2014 & $9.1(0.9)$ & $9.7(0.9)$ & $10.6(0.9)$ & $11.2(1.1)$ & \\
\hline 2015 & $10.1(1.0)$ & $9.8(0.9)$ & $9.1(0.8)$ & $13.7(1.2)$ & \\
\hline 2016 & $9.4(0.6)$ & $9.2(0.6)$ & $10.7(0.6)$ & $17.5(1.1)$ & \\
\hline Education (year) & & & & & $<0.001$ \\
\hline$\leq 6$ & $47.6(1.0)$ & $23.7(0.8)$ & $12.2(0.6)$ & $6.0(0.4)$ & \\
\hline $7-9$ & $20.3(0.8)$ & $19.7(0.8)$ & $14.2(0.7)$ & $8.1(0.5)$ & \\
\hline $10-12$ & $22.4(0.8)$ & $38.5(0.9)$ & $39.1(1.0)$ & $33.1(1.0)$ & \\
\hline$\geq 13$ & $9.7(0.6)$ & $18.0(0.8)$ & $34.5(0.9)$ & $52.9(1.1)$ & \\
\hline Marital status & & & & & $<0.001$ \\
\hline Single & $12.9(0.7)$ & $11.1(0.6)$ & $11.8(0.7)$ & $17.8(1.1)$ & \\
\hline Married & $87.1(0.7)$ & $88.9(0.6)$ & $88.2(0.7)$ & $82.2(1.1)$ & \\
\hline Residential area & & & & & $<0.001$ \\
\hline Urban & $66.8(1.6)$ & $74.6(1.4)$ & $80.6(1.2)$ & $85.1(1.2)$ & \\
\hline Rural & $33.2(1.6)$ & $25.4(1.4)$ & $19.4(1.2)$ & $14.9(1.2)$ & \\
\hline Current smoking & $53.0(1.0)$ & $52.2(1.0)$ & $50.6(1.0)$ & $43.7(1.0)$ & $<0.001$ \\
\hline Alcohol intake in past month & $68.7(0.8)$ & $79.6(0.7)$ & $83.2(0.7)$ & $86.3(0.7)$ & $<0.001$ \\
\hline Physical activity $^{\mathrm{a}}$ & $43.1(1.0)$ & $40.4(0.9)$ & $38.6(0.9)$ & $38.7(0.9)$ & 0.004 \\
\hline Health checkup in the prior 2 years & $56.6(1.0)$ & $64.2(0.9)$ & $70.5(0.9)$ & $78.6(0.7)$ & $<0.001$ \\
\hline Cancer examination in the prior 2 years & $46.6(0.9)$ & $52.8(0.9)$ & $56.0(1.0)$ & $66.1(0.9)$ & $<0.001$ \\
\hline Hypertension $^{\mathrm{b}}$ & $52.2(1.0)$ & $42.5(0.9)$ & $39.4(1.0)$ & $37.2(0.9)$ & $<0.001$ \\
\hline Diabetes $^{\mathrm{c}}$ & $23.9(0.9)$ & $16.9(0.7)$ & $13.9(0.7)$ & $12.7(0.6)$ & $<0.001$ \\
\hline Dyslipidemia $^{\mathrm{d}}$ & $14.9(0.7)$ & $15.6(0.7)$ & $15.8(0.7)$ & $19.3(0.8)$ & $<0.001$ \\
\hline Cardiocerebrovascular disease & $12.3(0.6)$ & $5.3(0.4)$ & $4.4(0.4)$ & $3.8(0.3)$ & $<0.001$ \\
\hline Other cancer & $5.8(0.4)$ & $3.4(0.3)$ & $2.5(0.3)$ & $2.2(0.3)$ & $<0.001$ \\
\hline
\end{tabular}

All values are given as estimated percentage(standard error); ${ }^{a}$, Physical activity was indicated as 'yes' when the subject walked for more than 30 $\mathrm{min}$ at a time and more than five times per week; ${ }^{\mathrm{b}}$, Hypertension was defined as systolic blood pressure $\geq 140 \mathrm{mmHg}$ or diastolic blood pressure $\geq$ $90 \mathrm{mmHg}$ or taking antihypertension medication; ${ }^{\mathrm{c}}$, Diabetes was defined as fasting serum glucose $\geq 126 \mathrm{mg} / \mathrm{dL}$ or taking insulin or oral diabetes medication; ${ }^{\mathrm{d}}$, Dyslipidemia was defined as taking a dyslipidemic medicine or total cholesterol $\geq 240 \mathrm{mg} / \mathrm{dL}$ or triglycerides $\geq 200 \mathrm{mg} / \mathrm{dL}$ or lowdensity lipoprotein cholesterol $\geq 160 \mathrm{mg} / \mathrm{dL}$ or high-density lipoprotein cholesterol $\leq 40 \mathrm{mg} / \mathrm{dL}$

SES and prevalent prostate cancer using 2007-2016 KNHANES data and the trends of the AIR and AMR in prostate cancer from 2000 to 2016 using cancer registration and cause-of-death data. In our results, 
Table 3. Characteristics of Subjects by Education Level

\begin{tabular}{|c|c|c|c|c|c|}
\hline \multirow[t]{2}{*}{ Variables } & \multicolumn{4}{|c|}{ Education (year) } & \multirow[t]{2}{*}{ P-value } \\
\hline & $\leq 6$ & $7-9$ & $10-12$ & $\geq 13$ & \\
\hline Prostate cancer patients & $0.5(0.1)$ & $0.2(0.1)$ & $0.2(0.1)$ & $0.4(0.1)$ & 0.015 \\
\hline Age (year) & & & & & $<0.001$ \\
\hline 40-49 & $7.5(0.6)$ & $20.1(1.1)$ & $44.8(0.9)$ & $57.4(0.9)$ & \\
\hline $50-64$ & $43.9(1.0)$ & $55.8(1.2)$ & $41.8(0.9)$ & $33.6(0.9)$ & \\
\hline$\geq 65$ & $48.6(1.0)$ & $24.1(0.9)$ & $13.4(0.5)$ & $8.9(0.4)$ & \\
\hline BMI $\left(\mathrm{kg} / \mathrm{m}^{2}\right)$ & & & & & $<0.001$ \\
\hline$<18.5$ & $4.6(0.4)$ & $2.4(0.3)$ & $1.8(0.2)$ & $1.6(0.2)$ & \\
\hline $18.5-24.9$ & $64.1(0.9)$ & $60.6(1.2)$ & $58.1(0.8)$ & $55.1(0.9)$ & \\
\hline $25.0-29.9$ & $29.2(0.9)$ & $34.2(1.1)$ & $36.6(0.8)$ & $39.5(0.9)$ & \\
\hline$\geq 30$ & $2.1(0.3)$ & $2.9(0.4)$ & $3.6(0.3)$ & $3.8(0.3)$ & \\
\hline Survey year & & & & & $<0.001$ \\
\hline 2007 & $5.9(0.8)$ & $5.4(0.8)$ & $4.5(0.6)$ & $3.9(0.6)$ & \\
\hline 2008 & $11.5(1.0)$ & $11.2(1.0)$ & $9.3(0.7)$ & $8.5(0.8)$ & \\
\hline 2009 & $11.9(1.0)$ & $11.4(1.0)$ & $9.8(0.8)$ & $9.5(0.9)$ & \\
\hline 2010 & $11.2(1.2)$ & $11.5(1.1)$ & $10.6(0.9)$ & $9.1(0.9)$ & \\
\hline 2011 & $10.8(1.1)$ & $11.8(1.2)$ & $10.8(0.9)$ & $9.8(0.9)$ & \\
\hline 2012 & $10.6(1.1)$ & $10.0(1.0)$ & $11.2(0.9)$ & $9.8(1.0)$ & \\
\hline 2013 & $9.6(0.9)$ & $10.3(1.0)$ & $11.2(0.9)$ & $10.5(1.0)$ & \\
\hline 2014 & $9.0(0.9)$ & $9.9(1.0)$ & $10.1(0.9)$ & $11.4(1.0)$ & \\
\hline 2015 & $10.1(1.0)$ & $8.3(0.9)$ & $11.2(0.9)$ & $12.0(1.1)$ & \\
\hline 2016 & $9.4(0.6)$ & $10.3(0.8)$ & $11.3(0.6)$ & $15.5(0.9)$ & \\
\hline Monthly household income & & & & & $<0.001$ \\
\hline Lowest & $44.1(1.0)$ & $25.0(1.0)$ & $12.1(0.5)$ & $5.8(0.4)$ & \\
\hline Medium-lowest & $31.1(0.9)$ & $34.3(1.2)$ & $29.4(0.8)$ & $15.2(0.7)$ & \\
\hline Medium-highest & $16.1(0.7)$ & $24.8(1.1)$ & $30.1(0.8)$ & $29.2(0.8)$ & \\
\hline Highest & $8.7(0.6)$ & $15.9(1.0)$ & $28.4(0.9)$ & $49.9(1.0)$ & \\
\hline Marital status & & & & & $<0.001$ \\
\hline Single & $9.8(0.6)$ & $11.3(0.8)$ & $13.7(0.7)$ & $17.0(1.0)$ & \\
\hline Married & $90.2(0.6)$ & $88.7(0.8)$ & $86.3(0.7)$ & $83.0(1.0)$ & \\
\hline Residential area & & & & & $<0.001$ \\
\hline Urban & $64.9(1.6)$ & $72.2(1.5)$ & $78.4(1.2)$ & $88.2(0.9)$ & \\
\hline Rural & $35.1(1.6)$ & $27.8(1.5)$ & $21.6(1.2)$ & $11.8(0.9)$ & \\
\hline Current smoking & $49.5(1.0)$ & $50.3(1.2)$ & $52.8(0.9)$ & $45.2(0.9)$ & $<0.001$ \\
\hline Alcohol intake in past month & $71.0(0.8)$ & $80.3(0.9)$ & $84.0(0.6)$ & $82.8(0.7)$ & $<0.001$ \\
\hline Physical activity ${ }^{\mathrm{a}}$ & $40.9(1.0)$ & $37.7(1.2)$ & $39.6(0.8)$ & $40.6(0.9)$ & 0.152 \\
\hline Health checkup in the prior 2 years & $62.2(1.0)$ & $64.0(1.2)$ & $67.9(0.8)$ & $76.0(0.8)$ & $<0.001$ \\
\hline Cancer examination in the prior 2 years & $51.5(1.0)$ & $55.4(1.2)$ & $54.5(0.8)$ & $62.0(0.9)$ & $<0.001$ \\
\hline Hypertension $^{\mathrm{b}}$ & $50.7(1.0)$ & $44.3(1.1)$ & $41.6(0.8)$ & $35.5(0.8)$ & $<0.001$ \\
\hline Diabetes $^{c}$ & $21.4(0.8)$ & $19.8(0.9)$ & $15.2(0.6)$ & $12.1(0.6)$ & $<0.001$ \\
\hline Dyslipidemia $^{\mathrm{d}}$ & $13.9(0.7)$ & $17.9(0.9)$ & $16.4(0.6)$ & $18.0(0.7)$ & $<0.001$ \\
\hline Cardiocerebrovascular disease & $10.2(0.6)$ & $8.7(0.6)$ & $4.8(0.3)$ & $3.1(0.3)$ & $<0.001$ \\
\hline Other cancer & $5.5(0.4)$ & $3.5(0.4)$ & $2.7(0.3)$ & $2.3(0.2)$ & $<0.001$ \\
\hline
\end{tabular}

All values are given as estimated percentage(standard error); ${ }^{a}$, Physical activity was indicated as 'yes' when the subject walked for more than 30 $\mathrm{min}$ at a time and more than five times per week; ${ }^{b}$, Hypertension was defined as systolic blood pressure $\geq 140 \mathrm{mmHg}$ or diastolic blood pressure $\geq$ $90 \mathrm{mmHg}$ or taking antihypertension medication; ${ }^{c}$, Diabetes was defined as fasting serum glucose $\geq 126 \mathrm{mg} / \mathrm{dL}$ or taking insulin or oral diabetes medication; ' , Dyslipidemia was defined as taking a dyslipidemic medicine or total cholesterol $\geq 240 \mathrm{mg} / \mathrm{dL}$ or triglycerides $\geq 200 \mathrm{mg} / \mathrm{dL}$ or lowdensity lipoprotein cholesterol $\geq 160 \mathrm{mg} / \mathrm{dL}$ or high-density lipoprotein cholesterol $\leq 40 \mathrm{mg} / \mathrm{dL}$.

prevalent prostate cancer increased significantly with household income and education level. Also, the AIR of prostate cancer increased sharply from 2000 to 2011 , while there was little change in the AMR between 2000 
Table 4. The ORs for Prevalent Prostate Cancer by Quartiles of Monthly Household Income and Education Level

\begin{tabular}{lccc}
\hline Variables & $\begin{array}{c}\text { Model 1 } \\
\text { OR }(95 \% \mathrm{CI})\end{array}$ & $\begin{array}{c}\text { Model 2 } \\
\text { OR (95\%CI) }\end{array}$ & $\begin{array}{c}\text { Model 3 }^{\mathrm{b}} \\
\text { OR (95\%CI) }\end{array}$ \\
\hline $\begin{array}{l}\text { Monthly household income } \\
\quad \text { Lowest }\end{array}$ & 1 & 1 & 1 \\
$\quad$ Medium-lowest & $1.88(0.90-3.91)$ & $1.95(0.93-4.09)$ & $1.87(0.90-3.87)$ \\
$\quad$ Medium-highest & $1.47(0.61-3.53)$ & $1.53(0.62-3.79)$ & $1.23(0.48-3.15)$ \\
$\quad$ Highest & $5.54(2.63-11.68)$ & $5.32(2.44-11.61)$ & $3.71(1.48-9.30)$ \\
Education (year) & & & 1 \\
$\leq 6$ & 1 & 1 & $0.67(0.19-2.35)$ \\
$7-9$ & $0.58(0.17-1.91)$ & $0.67(0.19-2.35)$ & $1.07(0.41-2.78)$ \\
$10-12$ & $1.00(0.40-2.53)$ & $1.08(0.42-2.79)$ & $3.66(1.54-8.70)$ \\
$\geq 13$ & $3.44(1.47-8.03)$ & $3.73(1.58-8.81)$ & \\
\hline
\end{tabular}

a, Adjusted by age, survey year, marital status and residential area; ${ }^{b}$, Adjusted by Model 1 variables plus BMI, current smoking, alcohol intake in past month, physical activity, health checkup in the prior 2 years, cancer examination in the prior 2 years, hypertension, diabetes, dyslipidemia, cardiocerebrovascular disease and other cancer; ${ }^{\circ}$, Adjusted by Model 2 variables plus education level or monthly household income level

and 2016.

In the present study, prevalent prostate cancer increased with SES, as evaluated using monthly household income and education level. In previous studies, the relationship between SES and prostate cancer was inconsistent. An analysis in 65,506 patients diagnosed with any cancer using Korean National Health Insurance cancer registration data showed that the high-income group had a 1.28-fold higher risk of prostate cancer than the low-income group (Kim et al., 2012), which is similar to our results. However, in a study of the relationship between income and prostate cancer among adults living in the US and Canada, the authors presented that prostate cancer increased with income in the United States, while income and prostate cancer were not related in Canada (Mackillop et al., 2000). The reason for this difference is that genetic and cultural factors and the medical systems, especially universal health coverage, differ from country to country (Liu et al., 2001). In addition, the association between SES and prostate cancer seems to be influenced by the introduction of the PSA screening test. In the United States, the incidence of prostate cancer was not associated with SES before the introduction of the PSA test. However, since then, the higher the SES, the higher the incidence of prostate cancer (Liu et al., 2001). Even in study conducted in Finland, where economic inequality is low and public health care is universal, the researchers demonstrated that more educated people underwent more PSA tests and had a higher incidence and lower mortality rate of prostate cancer than less educated people (Kilpeläinen et al., 2016).

Our finding that the prevalent prostate cancer increased with SES means one of the following. First, the risk factors for prostate cancer are increased in the high SES group, which actually increases the incidence and prevalence of prostate cancer. However, as described earlier, the risk factors for prostate cancer are still unclear (Platz and Giovannucci, 2006), and there is currently no evidence that the risk factors affecting only the high SES group have surged in recent decades in Korea. Second, the low SES group underwent less prostate cancer screening, leading to a higher mortality rate, which lowered the prevalence of prostate cancer in the lower SES group, suggesting a higher prevalence of prostate cancer in the high SES group. However, this hypothesis cannot explain our finding that the mortality rate of prostate cancer remained almost unchanged in the years 2000-2016. Finally, it is possible that the high SES group underwent more PSA screening and more prostate cancer was found than in

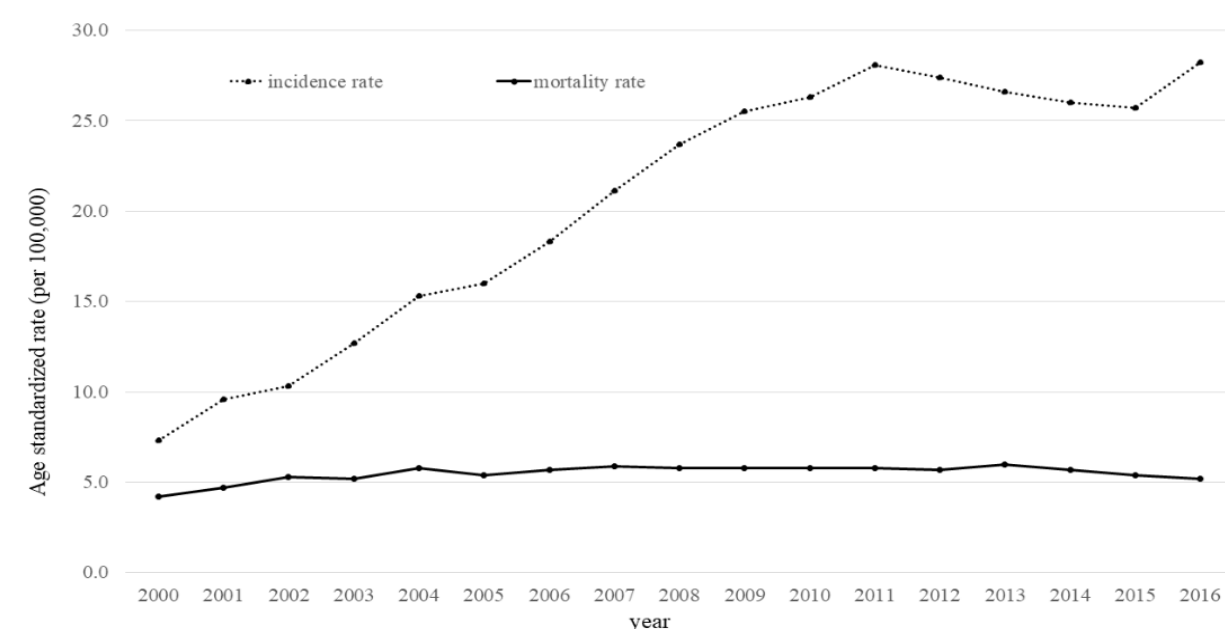

Figure 1. Age-Standardized Incidence and Mortality Rate of Prostate Cancer in 2000-2016 
the low SES group. Although the Korean national cancer screening program requires the entire Korean population to receive screening for five cancers (stomach, breast, colorectal, cervical, and liver) (Suh et al., 2016), prostate cancer screening is not included in this essential cancer screening program and additional personal expenses are required to receive prostate cancer screening (Kim et al., 2011). Therefore, the higher the SES, the higher the frequency of PSA testing, the greater the detection rate of prostate cancer, the better access to health care, and eventually the higher survival rate. As a result, the prevalent prostate cancer increases with a higher SES.

Our finding that prevalent prostate cancer increased with SES suggests overdiagnosis for several reasons. First, the incidence increased sharply, but the mortality did not change. This is a typical feature of overdiagnosis (Welch and Black, 2010) as seen in overdiagnosis of thyroid cancer in Korea. In addition, our results demonstrated that the incidence of prostate cancer has increased sharply from 2000 to 2011, and has suddenly decreased since 2012. Interestingly, from 2011, the media began to pay attention to overdiagnosis of thyroid cancer (Korea Times, 2014). Since then, the incidence of thyroid cancer in Korea has decreased significantly (annual percentage change in 1999-2011: 22.4, in 2011-2015: -14.4) (Jung et al., 2018), similar to the incidence of prostate cancer. These results suggest that the social impact of the overdiagnosis of thyroid cancer might similarly affect the incidence of prostate cancer. Second, the result is because of the nature of prostate cancer itself. Prostate cancer occurs frequently in men, but progresses very slowly and has a long survival period, so there is controversy over the effects and necessity of early screening (Etzioni et al., 2002). The autopsy results of people who died from causes other than prostate cancer found that $52 \%$ of patients over age 50 and $77 \%$ of patients over age 70 had prostate cancer (Hoffman, 2011). Third, as in the developed countries, the increase in prostate cancer in Korea is mainly due to an increase in PSA testing. Although PSA tests are mainly used as early screening for prostate cancer, there is little evidence that PSA tests can reduce prostate cancer mortality (Andriole et al., 2012). In a systematic review, the sensitivity and specificity of the PSA test were only $21 \%$ and $91 \%$, respectively, based on a level of $4 \mathrm{ng} / \mathrm{mL}$, suggesting that the PSA test is an incomplete screening tool (Wolf et al., 2010). In a follow-up study of PSA screening and control groups in the Prostate, Lung, Colorectal, and Ovarian (PLCO) cancer screening trial with 76,693 men, the PSA screening group had a higher incidence of prostate cancer, but prostate cancer mortality was not significantly different between the two groups (Andriole et al., 2012). The European Randomized Study of Screening for Prostate Cancer (ERSPC) observed the PSA screening group and control group at nine centers in eight countries for 13 years; the mortality rate of the PSA screening group was significantly lower in only two centers (the Swedish and Dutch centers), and there were no significant differences in the other seven centers (Schröder et al., 2014).

This study has several limitations. First, it was impossible to clearly present cause-and-effect relationships because a cross-sectional survey was used. Second, prevalent prostate cancer was estimated by referring to questionnaire data rather than medical records. Third, there were no data on tumor size, cancer stage, or histopathology.

In conclusion, prevalent prostate cancer increased significantly with household income and education level and the increase in prevalent prostate cancer in Korea may not be due to an actual increase in prevalence, but to early detection such as PSA screening.

\section{Acknowledgements}

None.

\section{References}

Ahn HS, Kim HJ, Welch HG (2014). Korea's thyroid-cancer "Epidemic" - screening and overdiagnosis. NEngl J Med, 371, 1765-7.

Andriole GL, Crawford ED, Grubb RL, et al (2012). Prostate cancer screening in the randomized Prostate, Lung, Colorectal, and Ovarian Cancer Screening Trial: mortality results after 13 years of follow-up. J Natl Cancer Inst, 104, 125-32.

Baquet CR, Horm JW, Gibbs T, et al (1991). Socioeconomic factors and cancer incidence among blacks and whites. J Natl Cancer Inst, 83, 551-7.

Borg V, Kristensen TS (2000). Social class and self-rated health: can the gradient be explained by differences in life style or work environment?. Soc Sci Med, 51, 1019-30.

Cheng I, Witte JS, McClure LA, et al (2009). Socioeconomic status and prostate cancer incidence and mortality rates among the diverse population of California. Cancer Causes Control, 20, 1431-40.

Choi SW, Ryu SY, Han Ma, et al (2013). The association between the socioeconomic status and thyroid cancer prevalence; based on the Korean National Health and Nutrition Examination Survey 2010-2011. J Korean Med Sci, 28, 1734-40.

Cronin KA, Lake AJ, Scott S, et al (2018). Annual report to the nation on the status of cancer, part I: national cancer statistics. Cancer, 124, 2785-800.

Etzioni R, Penson DF, Legler JM, et al (2002). Overdiagnosis due to prostate-specific antigen screening: lessons from U.S. prostate cancer incidence trends. J Natl Cancer Inst, 94, 981-90.

Global Cancer Observatory (2018). Cancer Today. International Agency for Research on Cancer/World Health Organization, Lyon, France. Available from: http://gco.iarc.fr/today/home. Accessed:28/Feb/2019.

Hoffman RM (2011). Clinical practice. screening for prostate cancer. $N$ Engl J Med, 365, 2013-9.

Jung KW, Won YJ, Kong HJ, et al (2018). Cancer statistics in Korea: incidence, mortality and survival in 2015. Cancer Res Treat, 50, 303-16.

Jung KW, Won YJ, Kong HJ, et al (2019). Cancer statistics in Korea: incidence, mortality and survival in 2016. Cancer Res Treat, 51, 417-30.

Jung KW, Won YJ, Park S, et al (2009). Cancer statistics in Korea: incidence, mortality and survival in 2005. J Korean Med Sci, 24, 995-1003.

Kilpeläinen TP, Talala K, Raitanen J, et al (2016). Prostate cancer and socioeconomic status in the Finnish randomized study of screening for prostate cancer. Am J Epidemiol, 184, 720-31.

Kim JM, Kim HM, Jung BY, et al (2012). The association between cancer incidence and family income: analysis of Asian Pacific Journal of Cancer Prevention, Vol $20 \mathbf{3 1 4 3}$ 
Korean National Health Insurance cancer registration data. Asian Pac J Cancer Prev, 13, 1371-6.

Kim SC (2004). A continuous increase in prevalence of prostate cancer in Korea and its causes. J Korean Med Assoc, 47, 394-402.

Kim Y, Jun JK, Choi KS, et al (2011). Overview of the national cancer screening programme and the cancer screening status in Korea. Asian Pac J Cancer Prev, 12, 725-30.

Korean Statistical Information Service (2018). Statistics Korea, Daejeon. Available from: http://kosis.kr/statisticsList/ statisticsListIndex.do?menuId=M_01_01\&vwcd=MT_ZT ITLE\&parmTabId=M_01_01\&statId=1997018\&themaId $=$ D\#SelectStatsBoxDiv. Accessed: 28/Feb/2019.

Korea Times (2014). What caused jump in thyroid cancer cases? Available from: http://www.koreatimes.co.kr/www/news/ culture/2014/03/319_154183.html. Accessed: 28/Jun/2019.

Kweon S, Kim Y, Jang M-J, et al (2014). Data resource profile: the Korea National Health and Nutrition Examination Survey (KNHANES). Int J Epidemiol, 43, 69-77.

Liu L, Cozen W, Bernstein L, et al (2001). Changing relationship between socioeconomic status and prostate cancer incidence. J Natl Cancer Inst, 93, 705-9.

Mackillop WJ, Zhang-Salomons J, Boyd CJ, et al (2000). Associations between community income and cancer incidence in Canada and the United States. Cancer, 89, 901-12.

Malik SS, Batool R, Masood N, et al (2018). Risk factors for prostate cancer: A multifactorial case-control study. Curr Probl Cancer, 42, 337-43.

Perez-Cornago A, Key TJ, Allen NE, et al (2017). Prospective investigation of risk factors for prostate cancer in the UK Biobank cohort study. Br J Cancer, 117, 1562-71.

Platz EA, Giovannucci E (2006). Cancer Epidemiology and Prevention, NewYork, Oxford University Press, 1128-50.

Saydah SH, Imperatore G, Beckles GL (2013). Socioeconomic status and mortality: contribution of health care access and psychological distress among U.S. adults with diagnosed diabetes. Diabetes Care, 36, 49-55.

Schröder FH, Hugosson J, Roobol MJ, et al (2014). Screening and prostate cancer mortality: results of the European Randomised Study of Screening for Prostate Cancer (ERSPC) at 13 years of follow-up. Lancet, 384, 2027-35.

Schwartz KL, Crossley-May H, Vigneau FD, et al (2003). Race, socioeconomic status and stage at diagnosis for five common malignancies. Cancer Causes Control, 14, 761-6.

Suh M, Choi KS, Park B, et al (2016). Trends in cancer screening rates among Korean men and women: results of the Korean national cancer screening survey, 2004-2013. Cancer Res Treat, 48, 1-10.

Welch HG, Black WC (2010). Overdiagnosis in cancer. J Natl Cancer Inst, 102, 605-13.

Williams RR, Horm JW (1977). Association of cancer sites with tobacco and alcohol consumption and socioeconomic status of patients: interview study from the Third National Cancer Survey. J Natl Cancer Inst, 58, 525-47.

Wolf AMD, Wender RC, Etzioni RB, et al (2010). American Cancer Society guideline for the early detection of prostate cancer: update 2010. CA Cancer J Clin, 60, 70-98.

This work is licensed under a Creative Commons AttributionNon Commercial 4.0 International License. 